\title{
Large Snake Size Suggests Increased Snakebite Severity in Patients Bitten by Rattlesnakes in Southern California
}

\author{
Donald N. Janes, Jr, PhD, MD; Sean P. Bush, MD; Gita R. Kolluru, PhD \\ Lynchburg General Hospital, Lynchburg, VA (Dr Janes); Loma Linda University Medical Center, Loma Linda, CA (Dr Bush); and California \\ Polytechnic State University, San Luis Obispo, CA (Dr Kolluru).
}

\begin{abstract}
Objective.-To correlate rattlesnake size and other characteristics of envenomation with the severity of envenomation.

Methods.-We retrospectively reviewed 145 charts of patients bitten by rattlesnakes in Southern California between 1995 and 2004, measuring Snakebite Severity Scores (SSS) and characteristics of envenomation that might be correlated with snakebite severity, including rattlesnake size, rattlesnake species, patient size, and anatomic location of the bite. Outcomes measured included SSS, complications of envenomation, number of vials of antivenom used, and length of hospital stay.

Results.-Of the patients bitten by rattlesnakes, $81 \%$ were men, and $79 \%$ of bites were on the upper extremities. Fifty-five percent of bites were provoked by the patient, and $44 \%$ were unprovoked. Neither location of snakebite nor provocation of snakebite affected the SSS. Only 1 patient had a snakebite without envenomation, and only 1 patient died from envenomation. Rattlesnake size was positively correlated with SSS, and SSS was positively correlated with the number of vials of antivenom used and with the length of hospital stay. Rattlesnake species and patient mass did not affect SSS.
\end{abstract}

Conclusions.-Larger rattlesnakes cause more severe envenomations, which contradicts popular belief.

Key words: rattlesnake, envenomation, severity, antivenom, venom, snakebite

\section{Introduction}

\section{BACKGROUND}

Rattlesnake bites account for the majority of snake envenomations in the United States, and cause multiple severe systemic and local tissue effects, including respiratory compromise, ${ }^{1-3}$ coagulopathy, ${ }^{4-6}$ necrosis, ${ }^{7}$ and compartment syndrome. ${ }^{8}$ The severity and specific clinical effects of envenomation can be quantified using the Snakebite Severity Score (SSS), ${ }^{9}$ which includes effects on the local tissue, cardiovascular, respiratory, gastrointestinal, hematologic, and neurologic systems. To our knowledge there have been no studies addressing physicians' ability to predict which snakebites will result in more or less severe envenomations, and there are few studies addressing the treatment and outcome of envenomations from different snake species and sizes.

Corresponding author: Donald N. Janes, Jr, PhD, MD, Lynchburg General Hospital, 1901 Tate Springs Rd, Lynchburg, VA 24501 (email: donjanes@earthlink.net).

\section{IMPORTANCE}

We hypothesized that there may exist snake and patient characteristics that correlate with the severity of envenomation and with the relative amount of antivenom required to treat symptoms and prevent recurrence or progression. For example, we know that increasing rattlesnake size is associated with increasing volume of venom injected in snakebites. ${ }^{10}$ A larger venom volume should correlate with a higher SSS, and require a larger amount of antivenom to neutralize its effects. Thus, being able to predict dosing requirements could maximize antivenom efficacy and optimize patient outcome.

\section{GOALS OF THIS INVESTIGATION}

Our primary goal was to establish the correlation between snake size and snakebite severity. We retrospectively reviewed medical records to identify aspects of rattlesnake envenomations, including patient character- 
istics and rattlesnake size, which may correlate with SSS and with the amount of antivenom used to treat the patients. We also noted the short-term and long-term outcomes for patients, including complete recovery, recovery with deficits, and patient death. In addition, because venom components differ among snake species (eg, Crotalus scutulatus venom may have a prominent neurotoxic component ${ }^{11}$ ), bites by different snake species may result in different clinical effects. We therefore determined the effects of snake species on the components of SSS.

\section{Methods}

\section{STUDY DESIGN}

We retrospectively reviewed the electronic medical records identified by the International Classification of Diseases, Ninth Revision (ICD-9) codes E905.0 (venomous snake and lizard bites), and 989.5 (toxic effect of venom), between 1995 and 2004. During these years one of the investigators (S.P.B.) was involved in the majority of the envenomation cases, and prospectively included estimated or measured snake sizes, species, and SSS in his patient encounter documentation. From 1995 to 2001, 15 patients were treated with equine-derived Crotalidae polyvalent antivenom from Wyeth-Ayerst (New York, NY) and these patients were excluded from usage analyses of $\mathrm{CroFab}^{\circledR}$ (Crotalidae Polyvalent Immune $\mathrm{Fab}$ [Ovine][Protherics US Inc, Brentwood, TN]). The protocol was reviewed by the institutional review board and considered exempt from informed consent.

\section{STUDY SETTING AND POPULATION}

This study was conducted at an academic hospital with an annual Emergency Department census of 50,000 to 60,000 visits, and a large area of referral for cases of envenomation. Patients were included if they were bitten by a rattlesnake and had some estimate of snake size included in the medical record. Patients were excluded if any animal other than a rattlesnake bit them or if they were not bitten (eg, 1 patient had an eye splash). Rattlesnakes are the only venomous snakes native to areas where the patients in the study population were bitten.

\section{DATA COLLECTION}

Abstractors included 2 investigators, neither of whom was blinded to the goals of the study, and both of who were trained by the attending physician (S.P.B.). A standardized abstraction form was used. Periodic meetings were held during the data collection period to check the performance of the abstractors, discuss coding rules, and resolve disputes. All 3 investigators reviewed 10 records and inter-rater reliability was measured using the kappa statistic. For calculating the SSS, each component required assessment of a patient's signs and symptoms. If there was no mention of an organ system abnormality in the history of present illness, review of systems, vital signs, or physical examination, then that system was assumed to be unaffected by the snakebite. For the remainder of data analyses, if data were missing the chart was excluded from that particular analysis.

\section{DATA ANALYSIS}

All data were transformed to meet parametric assumptions. SSS, fang wound spread, vials of $\mathrm{CroFab}^{\circledR}$ antivenom, and duration of hospital stay were log-transformed, and components of SSS were arcsin squareroot-transformed. All analyses were performed using JMP 3.2.2 (SAS Institute, Inc, Cary, NC) at a 2-tailed $\alpha$ of .05 . Due to the discontinuous distribution of snake sizes, we grouped snake size data into categories: small $(<40 \mathrm{~cm}$ snout-tail length; $\mathrm{n}=61)$, medium $(40-75 \mathrm{~cm}$; $\mathrm{n}=28)$, and large $(>75 \mathrm{~cm} ; \mathrm{n}=30)$. For 23 cases the snake size was unknown.

We examined the effects of bite location (upper vs lower extremity) and whether the bite was provoked by intentional interaction with the snake on SSS using analysis of variance (ANOVA). To evaluate the effects of snake size, snake species and patient mass on snakebite severity, we employed an analysis of covariance model, with SSS as the dependent variable, categorical snake size (small, medium, large) and snake species (Crotalus atrox, $C$ cerastes, $C$ helleri, $C$ ruber, $C$ scutulatus) as fixed effects, and patient mass as the covariate. $C$ mitchellii envenomations were excluded from this analysis because of the small number of bites by this species in our dataset.

We performed a single factor ANOVA with categorical snake size as the independent variable and patient mass as the dependent variable. We also examined the effects of snake species on the components of SSS by performing a series of univariate ANOVAs (with Bonferroni correction for multiple tests), with snake species as the fixed effect and component (local wound, pulmonary, neurologic, cardiovascular, hematologic, gastrointestinal) as the dependent variable.

The relationship between fang wound spread and snake size was examined using ANOVA, with categorical snake size as the fixed effect and fang wound spread as the dependent variable. We examined the relationship between snake size and outcome, and SSS and outcome using 2 measures: vials of $\mathrm{CroFab}^{\circledR}$ used and duration of 
hospital stay. In each case, the analysis of covariance model included a fixed effects snake species term and patient mass and SSS as covariates.

\section{Results}

\section{PATIENT CHARACTERISTICS}

We reviewed 145 charts; of these, 3 were excluded because no snakebite occurred. For each statistical analysis, some charts were excluded due to insufficient information; in each case, the number of charts that was excluded can be determined by the sample sizes (ie, by comparing each sample size to 142 initial charts). Male patients comprised $81 \%$ of the group, with an average age of 32 years (range, 1.2-97 years), and an average mass of $72 \mathrm{~kg}$ (range, $11-157 \mathrm{~kg}$ ). Twenty-five patients admitted to consuming alcohol at the time the bite occurred.

\section{SNAKE AND SNAKEBITE CHARACTERISTICS}

Our dataset includes bites from the following Crotalus species: $C$ atrox, $C$ cerastes, $C$ helleri, $C$ mitchellii, $C$ ruber, and $C$ scutulatus. Snake species and size were determined by visual examination of the snake (or a photograph of the snake) by the physician $(n=49)$, patient description combined with geographic location (n =94), and/or expert testimony if the patient was also a snake expert $(\mathrm{n}=1)$. Dr Bush was involved in the vast majority of species identifications. In cases where these methods appeared unreliable, data were excluded from analysis $(n=2)$. Only 1 patient with a credible rattlesnake bite had an SSS of 0, making the incidence of dry bites in this population $<1 \%$.

There was no significant relationship between snake size and patient mass (ANOVA, $\mathrm{F}=1.3, P=.28$.) Thirty bites were located in the lower extremities and 112 bites were located in the upper extremities. There was no difference in SSS between bites in these 2 locations (ANOVA, $\mathrm{F}_{1,139}=3.47, P=.06$ ). Patients provoked 78 bites (ie, the patient was intentionally interacting with the snake) and 62 bites were unprovoked. There was no difference in SSS between provoked and unprovoked bites (ANOVA, $\mathrm{F}_{1,137}=0.01, P=.92$ ). Four bites were due to fang punctures from dead snakes. One patient died from complications of envenomation. The Table lists other short-term complications from the snakebites and from antivenom.

\section{INTER-RATER RELIABILITY/REPEATABILITY}

Among the 10 charts reviewed by a third abstractor, there were no major discrepancies found in qualitative data.
There was perfect agreement on snake species $(\kappa=1)$, excellent agreement on snake size (weighted $\kappa=0.92$, $\mathrm{SE}=0.23)$, and excellent correlation of SSS $\left(r^{2}=0.98\right)$.

\section{FACTORS INFLUENCING SSS}

Snakebite severity increased with increasing snake size (ANCOVA, $\mathrm{n}=99, \mathrm{~F}_{2,83}=5.49, P=.006$; Figure 1). Independent contrasts revealed that there was a significant difference in SSS between small and medium snakes ( $t$ test; $t=2.45, P=.016$ ) and between small and large snakes $(t=2.87, P=.005)$ but not between medium and large snakes $(t=0.20, P=.84)$. Snakebite severity was not significantly influenced by patient mass $\left(\mathrm{F}_{1,83}=3.67, P=.059\right)$ or snake species $\left(\mathrm{F}_{4,83}=2.20\right.$, $P=.077)$. There was a significant interaction between snake size and snake species $\left(\mathrm{F}_{8,83}=2.87, P=.007\right)$ because the relationship between snake size and snakebite severity was not the same across snake species (Figure 2).

\section{SPECIES DIFFERENCES IN SSS COMPONENTS}

After Bonferroni correction for multiple tests ( $\alpha$ corrected $=.008)$, the snake species differed from each other in the local wound component of SSS (ANOVA; $\mathrm{F}_{4,103}=5.90, P=.0003$ ) but did not differ with respect to the other components: pulmonary $\mathrm{F}_{4,103}=3.12, P=$ .018 ; cardiovascular $\mathrm{F}_{4,103}=2.75, P=.032$; hemato$\operatorname{logic} \mathrm{F}_{4,103}=2.51, P=.046$; central nervous system $\mathrm{F}_{4}$, $103=2.79, P=.030 ;$ gastrointestinal $\mathrm{F}_{4,103}=1.81, P$ $=.13$. $C$ cerastes caused the greatest relative local wound severity and $C$ ruber the least.

\section{FANG WOUND SPREAD}

Fang puncture wound spread increased with increasing snake size (ANOVA, $\mathrm{n}=39, \mathrm{~F}_{2,35}=14.75, P<.0001$; Figure 3). A post-hoc Tukey's test revealed that the largest size class differed significantly from the other 2 size classes but that the smaller size classes did not differ from each other. There was no relationship between fang puncture wound spread and patient mass $(P=.33)$.

\section{SNAKE SIZE, SNAKEBITE SEVERITY, AND PATIENT OUTCOME}

There was a significant effect of snake size on duration of hospital stay $\left(\mathrm{F}_{2,83}=8.60, P=.0004\right)$. A post-hoc Tukey's test revealed that the largest snake size class was associated with the longest hospital stay, and that the other 2 snake size classes did not differ significantly from each other. There was no correlation between snake 
Table. Complications from Snakebites and Antivenom

\begin{tabular}{|c|c|c|c|c|c|c|}
\hline $\begin{array}{l}\text { Patient age } \\
\quad(y)\end{array}$ & $\begin{array}{l}\text { Patient mass } \\
\quad(\mathrm{kg})\end{array}$ & Snake species & $\begin{array}{l}\text { Snake size } \\
\quad \text { class }\end{array}$ & Provoked? & SSS & Complication \\
\hline \multicolumn{7}{|c|}{ Complications from snakebite } \\
\hline 50 & 90 & $C$ helleri & Large & No & 13 & Death \\
\hline 33 & 63 & Unknown & Unknown & Yes & 6 & Rhabdomyolysis \\
\hline 41 & 80 & $C$ helleri & Large & $\mathrm{Yes}^{a}$ & 6 & Rhabdomyolysis \\
\hline 14 & 85 & $C$ helleri & Large & No & 7 & $\begin{array}{l}\text { Rhabdomyolysis, compartment syndrome, } \\
\text { fasciotomy }\end{array}$ \\
\hline 39 & 80 & $C$ mitchellii & Large & Yes & 5 & Compartment syndrome, fasciotomy \\
\hline 28 & 86 & $C$ mitchellii & Unknown & No & 6 & Compartment syndrome, fasciotomy \\
\hline 23 & 80 & $C$ helleri & Large & Yes & 16 & Compartment syndrome, fasciotomy \\
\hline 60 & 80 & $C$ helleri & Small & No & 3 & Lymphedema \\
\hline 79 & 81 & $C$ atrox & Medium & Yes & 10 & Cellulitis \\
\hline 27 & 100 & C ruber & Small & No & 3 & Delayed multicomponent coagulopathy \\
\hline 46 & 90 & $C$ atrox & Small & Yes & 3 & Persistently low fibrinogen \\
\hline 26 & 70 & $C$ atrox & Small & No & 6 & Persistently low fibrinogen \\
\hline 27 & 66 & $C$ helleri & Large & No & 9 & Persistent perioral paresthesias \\
\hline 6 & 20 & $C$ helleri & Large & No & 13 & $\begin{array}{l}\text { Sensory loss; long term physical therapy } \\
\text { needed }\end{array}$ \\
\hline \multicolumn{7}{|c|}{ Complications from antivenom } \\
\hline 48 & 157 & $C$ helleri & Small & No & 1 & Chest pain with $\mathrm{CroFab}^{\circledR}$ \\
\hline 37 & 80 & C scutulatus & Small & No & 1 & Anaphylactoid reaction to $\mathrm{CroFab}^{\circledR}$ \\
\hline 43 & 85 & $C$ helleri & Medium & Yes & 10 & Anaphylactoid reaction to $\mathrm{CroFab}^{\circledR}$ \\
\hline 64 & 100 & $C$ helleri & Small & Yes & 3 & Anaphylactoid reaction to $\mathrm{CroFab}^{\circledR}$ \\
\hline 39 & 80 & C mitchellii & Large & Yes & 5 & Anaphylactoid reaction to $\mathrm{CroFab}^{\circledR}$ \\
\hline 32 & 89 & $C$ helleri & Large & Yes & 5 & Anaphylactoid reaction to Wyeth antivenom \\
\hline 40 & 79.5 & Unknown & Medium & Yes & 6 & Serum sickness from Wyeth antivenom \\
\hline 9 & 30 & $C$ atrox & Unknown & No & 6 & Serum sickness from Wyeth antivenom \\
\hline
\end{tabular}

${ }^{a}$ Fang scratch from dead snake.

size and the number of vials of $\mathrm{CroFab}^{\circledR}$ used $(P=.94)$. There was a positive relationship between SSS and both the number of vials of $\mathrm{CroFab}^{\circledR}$ antivenom administered $\left(\mathrm{n}=79, \mathrm{~F}_{1,70}=37.12, P<.0001\right.$, standardized $\left.\beta=.62\right)$ and the duration of hospital stay $\left(\mathrm{n}=92, \mathrm{~F}_{1,83}=15.24\right.$,

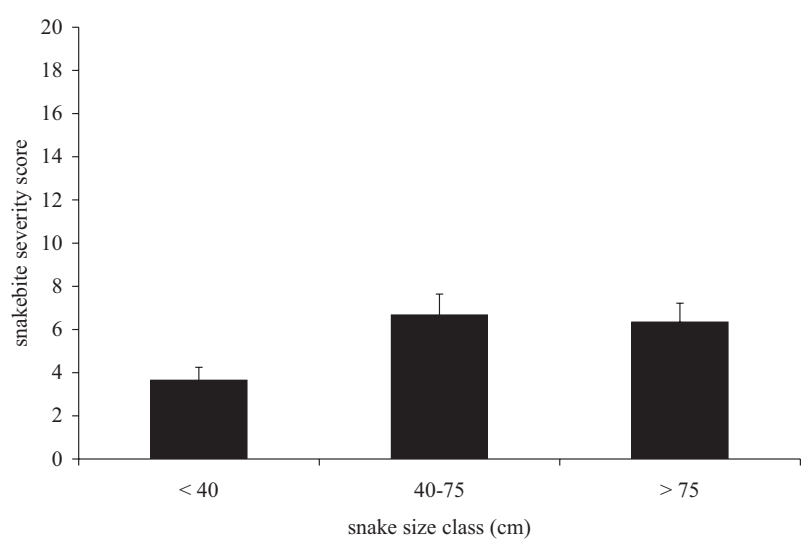

Figure 1. Mean snakebite severity scores from snakes of different size classes.
$P<.0001$, standardized $\beta=.39$ ). Mean number of vials of $\mathrm{CroFab}^{\circledR}$ used was 14 (range, 0-56) and mean hospital stay was 49 hours (range, 8 hours-15 days). These variables were not significantly influenced by snake species (both $P>.12$ ) or patient mass (both $P>.27$ ). Of 142 total patients, 40 patients were followed up within 42 days (median, 4.5 days), and 8 patients were seen as long

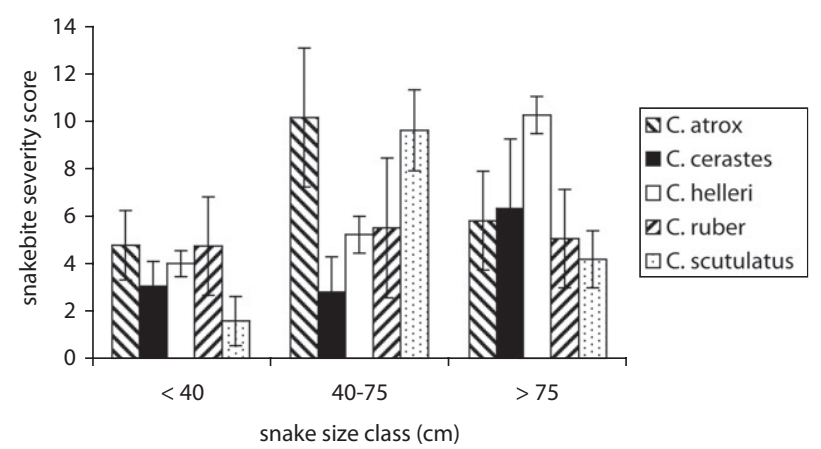

Figure 2. The effect of snake size class on snakebite severity score in different rattlesnake species. 


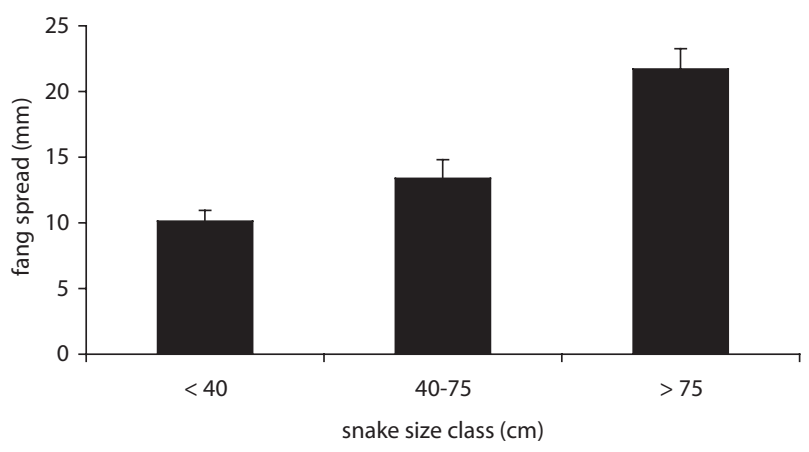

Figure 3. The relationship between fang spread (the space between fang puncture wounds) and snake size class.

as 1 to 6 years after the bite. Apart from the 1 patient who died, no other long-term complications were found in the medical records. One hundred thirteen patients received $\mathrm{CroFab}^{\circledR}$ antivenom, and five of these patients had acute reactions (Table).

\section{Discussion}

Ours is one of few studies to demonstrate that larger rattlesnake size correlates with greater snakebite severity. ${ }^{12}$ Popular belief often implies the opposite, that small snakes are more dangerous than larger snakes. Such a belief may have arisen because of speculation that younger snakes have less control over venom delivery and therefore deliver more venom (the opposite has been shown-larger, older snakes delivered an average of 17 times more venom in one study ${ }^{13}$ ) and because younger snakes have more toxic venom. ${ }^{14}$ Several authors have countered this belief with studies showing that larger snakes have more venom available ${ }^{15}$ and can cause more severe envenomations, ${ }^{12}$ and our study further supports this conclusion. Moreover, we showed that patients bitten by larger snakes and who had more severe envenomations received more vials of antivenom and had longer hospital stays, and that bites from larger snakes exhibited larger distances between fang puncture wounds. There have been few studies addressing optimal dosing of antivenom $^{16,17}$ and no such studies using CroFab ${ }^{\circledR}$; therefore, our study does not provide predictions of how much antivenom a given patient will ultimately require. However, we hope these data will help future investigators design studies to optimize antivenom dosing, and that eventually physicians may be able to judge how much antivenom to use based primarily on the size of the snake or the distance between the fang marks.

Children were not bitten by smaller snakes than adult patients, nor did smaller patients have more severe snakebites than larger patients. Our results are consistent with Wingert and Chan ${ }^{12}$ and with Tanen et al, ${ }^{18}$ the latter of whom found no difference between children and adults in the frequency of hematologic or local tissue derangements in their epidemiologic study of rattlesnake bites in Arizona. However, this remains surprising because one might predict that, on average, small children might have more severe envenomations than adults. ${ }^{7}$ There are data showing that rattlesnakes are able to meter their venom such that smaller prey may receive less venom than larger prey, ${ }^{19}$ and one could speculate that children, although they are not prey, may receive less venom than adult patients. On the other hand, there are also data showing that venom flow in defensive strikes does not differ with different target sizes, ${ }^{20}$ although arguably the mechanics of venom flow are not necessarily predictive of total venom delivery.

Although there was no difference in snakebite severity among snake species, there was a statistical interaction between snake size and snake species (ie, the snake species differed in the relationship between snake size and snakebite severity). This may have arisen as a sampling artifact as a consequence of small sample sizes; however, there may be a true difference among snake species in the relationship between snake size and snakebite severity. For example, sidewinders (C cerastes) exhibit a much smaller size range than most other species, and they are thought to have relatively weak venom. ${ }^{7,12}$ Therefore there may be much less difference in snakebite severity between small and large sidewinders than between small and large Southern Pacific rattlesnakes $(C$ helleri). On the other hand, by the same reasoning, we predicted a priori that some species would probably cause more severe envenomations than other species, because of the differences in venom toxicities. It is interesting that we did not find this to be the case.

Consistent with many previous studies, ${ }^{21,22}$ we found that the majority of snakebites occurred in young male adults, that the majority of bites were provoked (ie, true accidental bites were less common), that alcohol consumption may have contributed to some patients' decision to interact with the snakes, and that fatalities were rare. However, in contrast to some other studies, snakebites without envenomation ("dry" bites) were very rare. Russell found that $18 \%$ to $22 \%$ of bites were dry, ${ }^{7}$ and Young and Zahn found that $35 \%$ of bites were dry, ${ }^{20}$ while Tanen et al, like us, documented a low incidence of dry bites (3\%). ${ }^{18}$ The differences among studies may be due to different ways of judging snakebite severity. For example, the Young and Zahn study measured actual venom flow through the venom duct, ${ }^{20}$ whereas another used data collected from multiple other studies to make an estimate. ${ }^{7}$ In the present study and that of Tanen et $\mathrm{al}^{20}{ }^{20}$ the observed clinical effects of envenomation were recorded. The way that these effects are judged may 
differ from study to study, such that one investigator may view a fang puncture with mild limited erythema and pain to be a dry bite, whereas another investigator may score the same findings as mild envenomation (as we did.)

Serious complications of snakebite were uncommon, and occurred in only 14 patients (Table). Apart from 1 patient who died of an apparent cerebrovascular accident, no other patient had any permanent disability recorded in the medical records. Eight patients were seen 1 to 6 years after their snakebite, and although those visits were all for other medical problems, no lingering ill effects from their prior envenomation were documented. Four patients had fasciotomy done for compartment syndrome, consistent with prior studies illustrating that surgical management is rarely indicated. ${ }^{23}$ One patient developed cellulitis 1 week after envenomation, consistent with prior observations that infections are extremely rare. ${ }^{24}$ Eight patients had complications from antivenom, including anaphylactic and anaphylactoid reactions to $\mathrm{CroFab}^{\circledR}$ (Table). One patient was intubated, and all were treated successfully with usual treatments for severe allergic reactions. Five of 113 patients treated with $\mathrm{CroFab}^{\circledR}$ antivenom (4\%) had acute reactions, all of which were successfully treated without sequelae. This rate of adverse reaction is actually less than that reported previously. ${ }^{25}$

Using the number of vials of $\mathrm{CroFab}^{\circledR}$ given and the length of hospital stay as potential markers of snakebite severity, we found that both markers correlated well with SSS. Thus we conclude that a patient's initial SSS may be used to roughly predict resource utilization for that patient. Likewise we found that large snake size was associated with longer hospital stays, but interestingly and surprisingly we found that snake size did not correlate with the number of vials of $\mathrm{CroFab}^{\circledR}$ given. There are a number of potential reasons why we found no effect, including the nonstandardized way in which physicians gave antivenom, sometimes based on a regular loading dose followed by routine maintenance doses and other times giving an initial dose but reserving subsequent doses solely for progression of symptoms. Interphysician variability in medical care for similar conditions is actually an important contemporary topic in the debate on healthcare reform and treatment of snake envenomation is likely subject to the same phenomenon of variability.

In summary, larger rattlesnakes caused more severe envenomations, which in turn correlated with larger amounts of $\mathrm{CroFab}^{\circledR}$ antivenom used and longer hospital stays. However, we cannot predict how much antivenom would be required for a given envenomation. Snake species did not influence the severity of envenomation, despite previous in vitro studies showing differences in venom toxicity. Different snake species may cause different qualitative clinical effects of envenomation, but this finding needs further validation. Rattlesnake bites without envenomation were rare, as were fatalities and documented permanent disability from envenomation.

\section{LIMITATIONS}

Despite our attempt to maintain the highest possible methodological rigor, the limitations of this study include those typical of chart reviews, especially potential bias from incomplete data in some charts. For some analyses many of the charts were missing data (eg, fang puncture wound spread and snake size, relationship between SSS and number of vials of $\mathrm{CroFab}^{\circledR}$ used), which may have significantly altered the results. Patient masses were noted in the medical charts, but not whether the masses were measured or estimated. There may have been a systematic bias in that more severe snakebites may have received more attention by physicians than mild snakebites, and one of our investigators (S.P.B.) may have been more likely to be involved in those cases, resulting in better documentation than in very mild envenomations, for which he was not consulted. Similarly, there may have been a referral bias in that more severe snakebites are sent to our institution, which may decrease the measured fraction of "dry bites." Some data were obtained from patient descriptions or estimates (snake size and species), which introduced a potential source of error that could have affected the results. However, we used broad categories of snake size to reduce the effect of this error, and if the identification of snake species was doubtful we excluded that case from analyses in which species was a variable. Because southern California rattlesnakes often have specific habitats (eg, $C$ helleri lives mostly in chaparral and $C$ scutulatus in the Mojave Desert), and because rattlesnakes have many non-overlapping areas in their geographic ranges (eg, C. scutulatus is primarily in San Bernardino County and northward, while $C$ atrox is mostly in Riverside, San Diego, and Imperial counties), we felt confident about the vast majority of species identifications. Small sample sizes limited this study, as they limit most studies of snake envenomation, because it is a relatively rare medical problem in the United States. The analysis of the effects of snake species on the individual components of the SSS was especially limited by small sample sizes, as this was a complex analysis with multiple data cells. However, this analysis was not intended as a definitive study on the qualitative effects of envenomation but rather as a preliminary description of how these effects may be estimated. Finally, interpretation of long-term patient 
outcomes is very limited in this study, as it was not prospectively done and all long-term follow-up (ie, 1 or more years post-envenomation) was gleaned from the medical records of the few patients who presented later for other medical problems.

\section{Acknowledgment}

We thank Dr Thomas Schilling for his invaluable assistance as an abstractor for this chart review.

\section{References}

1. Brooks DE, Graeme KA, Ruha AM, Tanen DA. Respiratory compromise in patients with rattlesnake envenomation. J Emerg Med. 2002;23:329-332.

2. Kerns W, Tomaszewski C. Airway obstruction following canebrake rattlesnake envenomation. J Emerg Med. 2001; 20:377-380.

3. Hinze JD, Barker JA, Jones TR, Winn RE. Life-threatening upper airway edema caused by a distal rattlesnake bite. Ann Emerg Med. 2001;38:79-82.

4. Offerman SR, Barry JD, Schneir A, Clark RF. Biphasic rattlesnake venom-induced thrombocytopenia. J Emerg Med. 2003;24:289-293.

5. Bush SP, Wu VH, Corbett SW. Rattlesnake venominduced thrombocytopenia response to antivenin (Crotalidae) polyvalent: a case series. Acad Emerg Med. 2000;7: 181-185.

6. Boyer LV, Seifert SA, Clark RF, et al. Recurrent and persistent coagulopathy following pit viper envenomation. Arch Intern Med. 1999;159:706-710.

7. Russell FE. Snake Venom Poisoning. Great Neck, NY: Scholium International; 1983.

8. Gold BS, Barish RA, Dart RC, et al. Resolution of compartment syndrome after rattlesnake envenomation utilizing non-invasive measures. J Emerg Med. 2003;24: 285-288.

9. Dart RC, Hurlbut KM, Garcia R, Boren J. Validation of a severity score for the assessment of crotalid snakebite. Ann Emerg Med. 1996;27:321-326.

10. Hayes WK, Bush SP, Herbert SS, et al. Defensive bites by rattlesnakes (genus Crotalus): Venom expenditure, envenomation severity, and the importance of snake size [abstract]. Biology of the Rattlesnakes Symposium, Loma Linda, CA, January 15-18, 2005.
11. Rhoten WB, Gennaro JF. Treatment of the bite of a Mojave rattlesnake. J Fla Med Assoc. 1968;55:338-340.

12. Wingert WA, Chan L. Rattlesnake bites in southern California and rationale for recommended treatment. West J Med. 1988;148:37-44.

13. Hayes WK. Ontogeny of striking, prey-handling, and envenomation behavior of prairie rattlesnakes (Crotalus $v$. viridis). Toxicon. 1991;29:867-875.

14. Fiero MK, Seifert MW, Weaver TJ, Bonilla CA. Comparative study of juvenile and adult prairie rattlesnake (Crotalus viridis viridis) venoms. Toxicon. 1972;10:81-82.

15. Hayes WK. Factors associated with the mass of venom expended by prairie rattlesnakes (Crotalus $v$. viridis) feeding on mice. Toxicon. 1992;30:449-460.

16. Jorge MT, Cardoso JL, Castro SC, et al. A randomized 'blinded' comparison of two doses of antivenom in the treatment of Bothrops envenoming in São Paulo, Brazil. Trans R Soc Trop Med Hyg. 1995;89:111-114.

17. Tariang DD, Philip PJ, Alexander G, et al. Randomized controlled trial on the effective dose of anti-snake venom in cases of snake bite with systemic envenomation. J Assoc Physicians India. 1999;47:369-371.

18. Tanen DA, Ruha AM, Graeme KA, Curry SC. Epidemiology and hospital course of rattlesnake envenomations cared for at a tertiary referral center in central Arizona. Acad Emerg Med. 2001;8:177-182.

19. Hayes WK. Venom metering by juvenile prairie rattlesnakes, Crotalus v. viridis: effects of prey size and experience. Anim Behav. 1995;50:33-40.

20. Young BA, Zahn K. Venom flow in rattlesnakes: mechanics and metering. J Exp Biol. 2001;204:4345-4351.

21. Cowles RA, Colletti LM. Presentation and treatment of venomous snakebites at a northern academic medical center. Am Surg. 2003;69:445-449.

22. Morandi N, Williams J. Snakebite injuries: contributing factors and intentionality of exposure. Wilderness Environ Med. 1997;8:152-155.

23. Hall EL. Role of surgical intervention in the management of crotaline snake envenomation. Ann Emerg Med. 2001; 37:175-180.

24. LoVecchio F, Klemens J, Welch S, Rodriguez R. Antibiotics after rattlesnake envenomation. J Emerg Med. 2002; 23:327-328.

25. Dart RC, Seifert SA, Boyer LV, et al. A randomized multicenter trial of crotaline polyvalent immune Fab (ovine) antivenom for the treatment for crotaline snakebite in the United States. Arch Intern Med. 2001;161: 2030-2036. 